\title{
Notes sur la pensée religieuse des Minyanka du
}

\section{Mali}

Notes on the religious conceptions of the Minyanka of Mali

Jean-Paul Colleyn

\section{OpenEdition}

\section{Journals}

Édition électronique

URL : http://journals.openedition.org/span/112

DOI : 10.4000/span. 112

ISSN : 2268-1558

Éditeur

École pratique des hautes études. Sciences humaines

\section{Édition imprimée}

Date de publication : 1 septembre 1975

Pagination : 19-34

ISSN : 0294-7080

\section{Référence électronique}

Jean-Paul Colleyn, « Notes sur la pensée religieuse des Minyanka du Mali », Systèmes de pensée en Afrique noire [En ligne], 1 | 1975, mis en ligne le 02 juillet 2013, consulté le 23 avril 2019. URL : http:// journals.openedition.org/span/112 ; DOI : 10.4000/span.112 
NOTES SUR LA PENSEE RELIGIEUSE DES MINYANKA DU MALI

par Jean-Paul COLLEYN

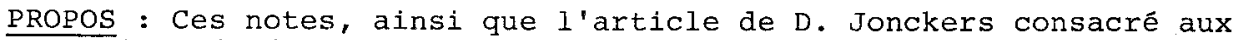
rites funéraires, sont le résultat d'une première approche de la vie religieuse des Minyanka. Le concept de religion appauvrit d'ailleurs quelque peu une pensée qui n'est pas seulement référence au sacré, mais surtout une logique du symbole. C'est à ce titre que la pensêe religieuse prend dans ses filets toutes les manifestations naturelles et culturelles, et assigne à chaque élément sa place. L'initiation religieuse étant basée sur le secret et 1 'apprentissage progressif, ces notes doivent être considérées à leur juste dimension : comme une étape de la recherche. N'ont été envisagés ici que les cultes (quelle que soit leur origine) considérés par les intéressés comme proprement minyanka, à l'exclusion des usages qu'ils déclarent empruntés à d'autres ensembles culturels.

Malgré une grande affinité culturelle avec les civilisations mandé, l'origine des Minyanka demeure incertaine. Deux types d'institutions structurent l'idéologie religieuse des Minyan$\mathrm{ka}$ : les cultes généraux, qui garantissent l'articulation harmonieuse du groupe et de son environnement et les sociétés d'initiation auxquelles on accède pour renforcer son statut social et parfaire ses connaissances. Dans le cadre des cultes initiatiques, la possession apparait comme un élément primordial de la communication avec le sacré : la "sortie" des autels nécessite l'intervention du ou des possédés qui, transmettant la parole des dieux qu'ils incarnent, jouent véritablement le rôle de médiums. Les principales figures du panthéon minyanka sont : Kle, Bieu créateur distant du monde des hommes, le Nya, gardien des valeurs, dieu de la fécondité et "tueur de sorciers", la hyène Sandsngs, patronne de l'agriculture et monitrice du monde, le Manỹa, "mère" de tous les cultes de possession au matériel rituel imposant. 


\section{A. INTRODUCTION.}

La tradition mandé rend compte d'une parenté mythique entre un grand nombre de peuples d'Afrique occidentale (1). Les Minyanka sont rattachés à une des familles alliées à la famille de Soundiata Keita.

L'origine mandé des Minyanka demeure cependant incertaine tant que l'histoire du peuplement n'aura pas été reconstituée.

Il est d'ores et déjà hors de doute que certaines familles dominantes du nord du pays Minyanka,sont d'origine malinké et marka.

Sur le plan linguistique, alors que du Sénégal au Bani une langue à peu près unique est parlée, on se heurte en territoire minyanka et senoufo à un ensemble souvent rattaché aux langues voltaiques. (2)

En dépit d'une parenté linquistique certaine entre les langues minyanka et senoufo, il faut remarquer que le poro, institution religieuse centrale du monde sénoufo, n'existe pas chez les Minyanka alors que le kore, société d'initiation bambara, se retrouve dans de nombreux villages minyanka.

En outre, de nombreuses institutions religieuses minyanka mettent en oeuvre des principes très proches de ceux de la philosophie religieuse bambara telle qu'elle a êté décrite par G. Dieterlen, D. Zahan et Y. Cissé.

La religion minyanka est absolument monothéiste. Le Dieu suprême, $K L \varepsilon$, domine toute la création qui procède de la vibra-

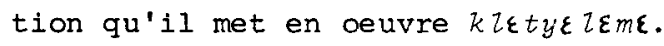

La cosmogonie constitue actuellement un vaste patrimoine éparpillé, que l'on ne peut reconstituer que par bribes. Elle met cependant en oeuvre des classifications "vécues quotidiennement" par l'ensemble de la population. KIE est une figure

(1) Voir surtout G. Dieterlen - Mythe et Organisation sociale en Afrique Occidentale, Journal de la Soc. des Afric., T.XXV, 1-2, 1955, p. 119 et T.XXIX, 1, 1959, p. 119 et D. Zahan, Aperçus sur la pensée théogonique des Dogon, Cahiers Internationaux de Sociologie, faris, vol. VI, 1949, p.113.

(2) Voir Baumann et Westermann, Les Feuples et les Religions d'Afrique; De Lavergne de Tressan, Inventaire linguistique de I'A.O.F., et du Togo, mém. IFAN $\mathrm{n}^{\circ} 30,1953$. 
lointaine, distante du monde des hommes, il est invoqué à l'occasion de toutes les prières, sauflors des pratiques de sorcellerie sikama, mais c'est généralement à des puissances inter médiaires entre Dieu et les hommes et sur lesquelles on a prise, que $I^{\prime}$ on s'adresse.

Il existe cependant dans chaque village, dans 1'axe de la plus ancienne porte, un autel en pisé portant le nom de $K$ L $\varepsilon$ et renfermant des fragments de l'environnement naturel et des premières installations humaines. Le descendant du fondateur $y$ fait, chaque année, au nom de tout le village, des sacrifices de poulets blancs; le blanc étant la couleur divine.

En construisant $1^{\prime a u t e} 1 K Z \varepsilon$, les fondateurs d'un village demandent à Dieu que, de Ia même façon qu'il a créé le monde, il permette aux hommes de créer le village. A ce titre, il constitue le premier des cultes généraux.

\section{B. LES CULTES GENERAUX.}

Deux types d'institutions structurent l'idéologie reliqieuse des minyanka.

La première catégorie est constituée par des cultes dont au moins un existe dans toute agglomération et qui imprèonent la vie villageoise. Ces institutions témoignent de l'articulation harmonieuse du groupe humain et de l'environnement naturel.

Puissants facteurs de cohésion au niveau villaquois, elles sont également garantes de l'ordre social. C'est sur leurs autels que seront offerts les sacrifices de réparation en cas de violations d'interdits remettant en cause l'ordre social: inceste, rixe mortelle au sein du villaque et autres actes considérés comme une "souillure de la terre".

\section{1) L'enclume tumpungo}

L'enclume du forgeron est considérée comme le réceptable de la plus grande et de la plus ancienne force, nyama. Elle est associée à l'aube de l'humanité et tous les principes sacrés s'inclinent devant elle, comme en témoignent les paroles de cette chanson à l'enclume recueillies à Kassorola : 
"Si personne ne peut plus rien, tumpungo le peut.

Le feu des sorciers est une simple trace à côté dé celui

de tumpungo.

C'est le tumpungo qui connait les remèdes de la pleine nuit. Ceux qui connaissent le mieux le secret des eaux n'ont qu'a aller chez les somono, ceux qui cherchent le yaperelautel portatif) le plus fort n'ont qu'à aller chez la tête de tous les yap\&re, le tumpungo".

L'enclume porte le nom du vautour tumpungo.

Selon les récits mythiques, c'est le vautour, symbole de la plus vieille connaissance, celle de "I'homme au crâne pelé", qui. a donné l'enclume aux hommes par l'intermédiaire du gbõngo (cynocéphale), le premier forgeron.

2) Le kãchys (littêralement la "jarre du village").

Le $k \tilde{a} c h y s$ est inséparable du rituel de fondation du quartier ou du village.

Souvent appelé "vieille mère", il témodgne des rapports harmonieux qui se sont établis entre les hommes comme entre les hommes et le milieu, lors de la fondation de l'aquglomération. Son culte, qui intéresse toute la communauté, qarantit la perpétuation de ces rapports.

L'élément principal de l'autel est une jarre, qui contient notamment des parcelles de l'environnement nature 1 auxque 1les ont été jointes des reliques des ancêtres. Les rites cycliques sont annuels, mais tous les sept ans les cérémonies revêtent une solennité particulière.

Les récits mythiques rendent compte d'une origine préhumaine du $k$ ăchys, institution donnée elle aussi aux hommes par le singe gbõngo.

3) Tyelq (littéralement "vieille femme".)

Le thème de la "vieille mère" est très important chez les Minyanka, il s'exprime surtout par un culte rendu à la première femme de la création.

Ce culte, parfois confondu avec le kãchyo est empreint d'une grande solennité, mais dans la littérature orale, Tye $\varepsilon_{\varepsilon}$ ou 
TyelEr symbolise la connaissance ambigüe, à la fois bonne et mauvaise ; "sa connaissance, dit-on, est celle des sorciers" sikãfo.

Le sanctuaire de Tyele se présente sous la forme d'une hutte circulaire dont le mur est constitue de rondins plantés verticalement dans le sol. Ces pièces de bois, qui soutiennent le toit de chaume, symbolisent les hommes du village qui soutiennent Tyez\&. L'animal sacrificiel par excellence est aujourd'hui le chien, mais selon les vieux d'ourikela, le sacrifice à Tyež était jadis un sacrifice humain.

4) Kachikãa.

Pour chaque village, il y a, sans compter les bosquets des cultes initiatiques, un bois sacré particulièrement touffu qui abrite les forces associées à la terre.

Les abords du bois sont soigneusement entretenus par les jeunes gens, groupés en société de culture, car si un feu de brousse venait à gagner le bois, les villageois n'auraient plus qu'à émigrer.

Le prêtre du kachikă est le chef de la terre, ningefs to, l'aine des descendants vivants de l'ancêtre qui noua un premier lien sacré avec le sol sur lequel réside le groupe.

Le chef de terre est souvent distinct du chef politique, dont le pouvoir, plus étendu mais plus fragile, est toujours contestable. Dans le domaine religieux, l'expression $k u l u f \jmath$ io, chef de village, désigne toujours le chef de terre.

Seuls, les chefs des familles très anciennes dans le village ont le droit de pénétrer dans le bois sacré à l'occasion d'un rite de réparation ou des sacrifices annuels. Ceux-ci ont lieu avant l'hivernage et aucun chef de famille ne manquerait d'offrir un poulet, puis un bouc ou un mouton au Kachika avant la nouvelle saison agricole.

(1) Nous n'avons pu établir avec certitude l'éthymologie de ce terme, que la plupart de nos informateurs traduisent par "bois sacré". 
La viande des animaux sacrificiels ne peut être consomiee gue par les habitants du viljage, leurs filles mariées et ies enfants de celle-ci. Elis ne sera jamais offexte à un stranger.

5) Le Dasiri.

Cette expression bambara, que les Miriyanka ont Iaraement adoptée, signifie littéralement "attacher la bouche". Fille s'emploie pour designer n'importe quelle pratique "maquique" destinśe à nuire à quelqu'un ou à en prendre le contrôle. L'expression s'applique donc également aux rites souvent liés à la fondation du village et qui visent à en assurer la protection et la perpetuation. Il s'agit "d'attacher la bouche des ennemis du village".

A ce titre, un animal auquel il ne sera fait aucun mal, rui sera nourri et jouira d'une liberté totale, symbolisera la permanence et la sérénité du villag̣e.

L'animal dasiri est le correspondant animal du kulufo 20 , au sens de représentant de l'ancêtre fondateur. Lors du rituel de fondation d'un quartier de Kimparana, le médium d'une société d'initiation, le Nya, possédé par la kãahyo est venu déclarer : "Koduba! (déformation de Koreduga, "vautour du kore", exclamation qui, chez les Minyanka, signale la manifestation d'une divinite) ninya na tyele djo na ye aujourd'hui ma vieille dire vous

yatıgo yala ye kere ya kayi le kulufozo"

animal chercher le laisser quartier chef de villace "Koduba! ma vieille mère (le kăchys est "mère" de tous les yaprre du village) m'a dit que vous deviez chercher un animal et le laisser au village comme chef de village".

Il y a une grande variéte d'animaux dasiri : un bouc à Nampossela, un âne à Ourikela et Yorosso, un chien noir à sanquela, un caïman à Ntiesso, des tortues à Somasso et M'petiona, des serpents à $M$ 'pessoba etc...

Au contraire du rite bambara, le kuludasiri (dasiri du village) ne sera jamais sacrifié. 
A la mort de l'animal, un "héritier" sera désigné par le devin tyєmfo (littéralement : maitre de la connaissance), consulté par le chef de village.

6) Fari.

Faro, le gênie de l'eau est bien connu des auteurs qui ont etudié 1' "univers" religieux bambara (1).

Chez les Minyanka, un culte régulier n'est rendu à Fari, qui apparait sous la forme du lamantin, qu'aux lieux d'éplphanie : il n'existe que dans les villages sur le territoire desquels se trouvent des rivières ou des marigots. Le descendant du fondateur y fait des offrandes annuelles de crème de mil et de lait, mais jamais de dolo ("dont Fari a horreur") ni de sacrifice sanglant. La colère de Fari est extrêmement redoutée et la récente sécheresse dont a souffert la réọion, a déterminé une recrudescence des rites liés au génie de l'eau.

Fari est également un thème mythique important et le fleuve Niger représente le corps de Nyadjopimakłrs "l'homme à la barbe en forme de pirogue", avatar de Fari qui s'est métamorphosé à l'issue d'une lutte avec ${ }^{\prime}$ ye $l \varepsilon r \varepsilon$, la première femme du monde.

(1) voir notamment G. Dieterlen, Essai sur la Religion bambara, Bibl. de sociologie contemporaine, Paris, PUF, 1951; $\mathrm{S}$. de Ganay, notes sur Ia Theodicée bambara in Revue de 2'Histoire des Religions, Paris t. $\operatorname{CXXXV}, \mathrm{n}^{\circ} 2-3$, avriljuin 1949, p. 187 ; Tauxier, La Religion bambara, Paris, Librairie orientaliste P. Geuthner, 1941 ; D. Zahan, Société d'initiation bambara, Mouton et Co, Paris-La Haye, 1960. 


\section{LES SOCIETES D'INITIATION.}

Parallèlement aux cultes genéraux qui participent à l'articulation du village avec le milieu environnant (forge, jarre du village, arbres sacrés, dasiri, Fari), existent des institutions qui trempent davantage dans l'organisation sociale au sens empirique du terme et que l'on regroupe sous le terme de dyo. Ce terme désigne à la fois I'initiation, les formules de consécration, le culte et la sociêté à laquelle on accède pour renforcer son statut social et parfaire ses connaissances. Quoique les dyo soient dépositaires d'un savoir ésotérique accessible aux seuls initiés, ils concernent toute la communauté et apparaissent comme les gardiens de l'ordre social. Ces cultes - à l'exception du kori, dont nous ne pouvons rendre compte ici - comportent un certain nombre d'éléments invariants :

1 - Un sanctuaire $g b a$, situé soit à l'intérieur, soit à l'extérieur du village.

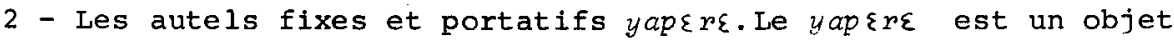
complexe, dont on dit qu'il est "dur" et charaé de forces d'autant plus redoutables qu'il est ancien et a reçu de nombreux sacrifices sanglants. Par extension, le terme yop $\varepsilon r \varepsilon$ désigne la puissance à laquelle le culte est rendu.

3 - Des instruments de musique qui sont spécifiques à chaque société.

4 - Un bois sacré ou plus souvent un enclos sacrificiel t $\tilde{u}$ situé à l'extérieur du village.

5 - Les sociétaires, parmi lesquels on distinque :

a) les "maitres" du culte (yaperefo)

b) les possédés (yapqr£ tutumo, littéralement "envoyé du y ap $x \boldsymbol{x}^{\prime \prime)}$,

c) les bouffons ( $y a p \varepsilon r \varepsilon$ koduba), initiés dont. la grande connaissance leur permet de tourner toutes choses en dérision. Quoique le terme soit une deformation du bambara . koreduga, "vautour du kore", étymologie que de nombreux Minyanka ignorent, chez les Minyanka, il existe, indépendemment de cette catécorie d'initiés du kori, des koduba qui n'entretiennent aucune relation particulière avec le kori et constituent une catégorie spécifique d'initiés au sein de chaque confrérie religieuse. 
d) les musiciens et chanteurs ( $y$ ap $r$ re ty $\varepsilon l i$ ) : quoique le terme dérive du bambara dye $z i$, qui désigne les membres d'une caste particulière, 11 designe, chez les Minyanka, des specialistes non castés dont le statut n'est pas différent de celui des autres membres de la société.

e) le sacrificateur, (mufo, litteralement, le "martre du couteau").

f) la yapere lф kachy : la femme donneuse d'eau. Elle rafrafchit les possédés et se charge des travaux ménagers en rapport avec 1 'exercice du culte.

g) les inities (yapere kude).

Pour la plupart des societés d'initiation, il $y$ a deux manifestations annuelles importantes ( $y$ ap $\varepsilon r \varepsilon$ kachi') qui se deroulent l'une avant les semailles, entre le debut du mois de mars et la fin mai, l'autre après les récoltes, à la fin du mois de novembre, début décembre. La plus importante de ces cerémonies comporte une réfection rituelle du sanctuaire.

Ces deux mantfestations annuelles se déroulent selon le schéma suivant :

Vers la fin de la matinée, le yapere s'empare du possédé. Celui-ci entre en transe et sort les autels portatifs du sanctuaire, en marchant à reculons, à l'instar des morts dans le monde desquels il se trouve de par la possession.

Il est assisté et encourage par les koduba et les tye $l_{i}$. La foule se fait de plus en plus nombreuse, tout le monde danse et reprend en choeur les chansons des tycli. Bientôt, un cortège se forme et entame une progession très. lente vers 1 'enclos artificiel ; les koduba s'efforcent par leurs faceties de freiner la marche pour ne pas "fatiguer le possede".

Celui-ci s'arrête à chaque lieu d'epiphanie : devant le sanctuaire d'un autre culte important, devant l'enclume des forgerons, devant la plus vieilie porte du village, qu'il franchit en marche arrière, enfin à l'extérieur du village, au premier carrefour. De temps en

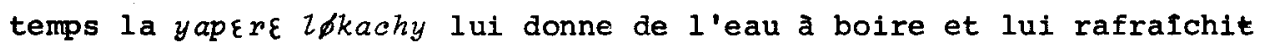
le visage. 
Au cours de cette procession, le possede communique les conseils, les remontrances et les menaces de la divinité. Très souvent, celleci se livre à une démonstration de force et quelqu'un, dans la foule, tombe victime d'une crise de courte durée mais très violente. Il reçoit aussitôt I'assistance des koduba.

Enfin, le cortège arrive à l'enclos sacrificiel, à proximité duquel la foule s'arrête.

Le possédé dépose les yapere à l'intérieur de l'enclos et les rites sacrificiels sont effectues en secret par les grands initiés. Les autels recevront ensuite, pendant l'après-midi, le sang des sacrifices propitiatoires effectués par le mũfo au nom des habitants du village.

A la tombée de la nuit, le yap rę "s'empare" à nouveau de son tutumo et la procession se forme dans l'autre sens, du yaper\&tũ vers le sanctuaire où le possédé doit ramener les autels portatifs.

A l'entrée du sanctuaire, il pose son fardeau et, jouant véritablement le rôle de médium, il réaffirme avec force la validité des coutumes traditionnelles et rappelle à chacun "son chemin", c'esta-dire son devoir et ses obligations vis-à-vis de la communauté. Après quoi, ont lieu les rites de rentrêe des autels qui précèdent les rejouissances.

1. Le (Nya) ou (Wara).

Les deux appelations sont interchanaeables, mais l'emploi du terme Wara domine chez les Minyanka du cercle de San, alors aue le terme Nya est préféré dans le reste du minyankala.

Quolque le lya soit souvent considéré comme un culte spécifiquement minyanka, il n'est pas exclu qu'il ait une oriaine mandé. Chaque Nya a un nom qu'il tient généralement d'une autre sociêté initiatique qui a présidé à sa consécration.

C'est ainsi que dans le cercle de Koutiala, les chefs de Nya TJksrmanya affirment que les anciens du village de Tokorla ont apporté les objets du culte du mandé.

Viviane Paques (1), mentionne le Nya parmi les cultes Bambara. 


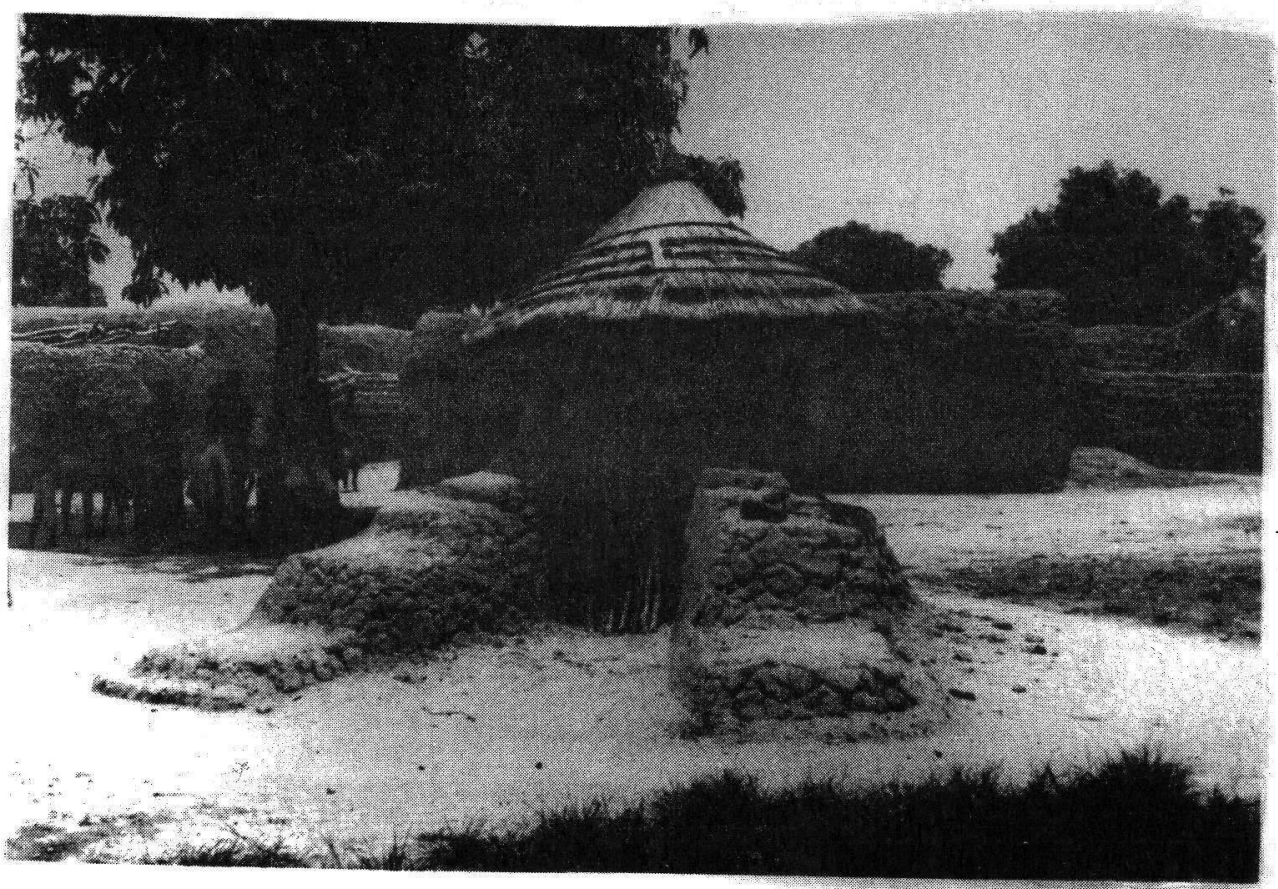

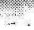

Sanctuaire du Nya du village de Gouantara (Mali) 
Les autres cultes qu'elle évoque (Komo, Kworè, Nama, Kono, Nago) sont d'ailleurs également connus des Minyanka.

Ailleurs (1), le même auteur nous livre une très brève description du Wara, autel propre aux cultivateurs sur lequel on sacrifie spécifiquement des chiens, et qui correspond à celle du wara minyanka.

Le terme bambara Wara signifie fauve, bête sauvage, animal griffu.

Selon une tradition recueillie en deux villades fort distants l'un desi'autre, Kimparana et Nkontina près de Koutiala, I'appelation Wara désigne le singe rouge Wara ble, (en minyanka : kstuns) qui a donné le Nya aux hommes.

Le lya protège le village ou le quartier; il est le garant de la moralité publique et plus particulièrement de celle des sociétaires, lesquels sont unis par une grande solidarité. Il sanctionne la violation des lois ancestrales. Jadis, le trésor accumulé par les sociétaires permettait de s'assurer la protection d'un "chef de guerre" puissant, de venir en aide aux villageois en difficulté et éventuellement, de racheter un membre de la société emmené en captivité à la suite d'une expédition ennemie. Les autels du Nya, répartis en trois sacs de toile de coton, se composent d'éléments divers qui, dans leur ensemble, constituent un véritable microcosme, siège de "toutes les forces du monde". Ces éléments renvoient, soit par métaphore, soit par métonymie à des créatures et des objets, eux-mêmes considêrés comme des symboles des grandes forces cosmiques. Ils peuvent faire l'objet d'une interprétation dont la complexité est a la mesure des connaissances de l'initié.

Les éléments constitutifs des autels admettent un classement en deux grandes catégories : celle des éléments marqués du signe positif, qui ont une influence favorable, et celle des éléments marqués néquativement, qui sont destinés à attaquer, à détruire. Cependant, selon les informateurs, cette classification est trop simple car certains principes - qui sont loin d'être les moins importants - se situent dans 1'ambiguité, à commencer par les

(1) V. Paques. Bouffons sacrés du Cercle de Bougouni. Journal de la Société des Africanistes, t. XXXIV, 1, 1964, 63. 
symboles de la connaissance elle-même.

Volci quelques elements constitutifs des yapere du Niya que nous avons pu identifier (1).

- griffe de lion tchãga: symbole de puissancé, de colère agressive.

- bec de vautour tumpungo: symbole de la "vieille connaissance". Ie vautour est un animal très vieux, au crâne degarni. Il est également immortel car, dit-on, il fait son nid et $y$ entre pour $y$ rester de longues semaines, avant d'en ressortir rajeunt, sous forme de ses jeunes dejà adultes.

- tête de grue couronnée gani : la grue couronnée est le symbole de la parole, de la connaissance humaine. C'est un oiseau divin, évoquant la fierté et l'orơueil bien placé.

- tête de cigogne masagǩgદ, littéralement "à côté de Dieu" : la cigogne est I'envoyé de Dieu; elle annonce la pluie, l'hivernage, la prospérité et $1^{\prime}$ abondance des récoltes. Elle est doublement bénefique. du fait qu'elle manque les sauterelles dévastatrices.

- tête de coq de brousse sekituntun, littéralement "le forgeron de brousse" : associé, comme son nom l'indique, à la forge et aux secrets de la technique metallurgique. Le Nya contient d'ailleurs une enclume miniature, rappel du yapere tumpungo que nous avons déjà évoqué. L'enclume, dit-on peut "monter jusqu'à Dieu en traversant les flammes".

- tête de calao segbaga ou tugbaga, en bambára, dyugó. Le calao est symbole de malheur. Quand on se promène en brousse et qu'on aperçoit un calao immobile, il faut lui jeter une pierre, le faire voler pour qu'on voie le bout de ses ailes blanc. Dans les incantations au $N y a$, on dit : "Faites que la tête de mon ennemi se courbe, comme la tête de calao". Il faut noter qu'une autre sorte de calao, (KIEtدgد), est considere comme un oiseau divin.

(1) cette presentation n'implique pas de notre part l'affirmation que les elements peuvent avoir une signification intrinsèque: elle correspond a l'ordre d'exposition adopté par les informateurs qui ont bien voulu prendre en charge notre initiation religieuse. 
Ie Nya comporte egalement des armes miniatures qui sont le symbole de la guerre victorieuse, celle qu'il mène contre les sorciers : "le $N y$ a est comme un arc tendu et comme la fleche qui penetre, 11 tranche comme le sabre". Ses autels contiennent egalement une charne en cuivre qui permet "d'attacher" les principes du mal. Parm. les yapere ou comme partiesconstitutives de ceux-ci, on trouve également les nombreuses racines ou autres éléments végêtaux, qui font partie de la pharmacopée traditionnelle. Ces racines n'ont pas seulement des vertus curatives, elles sont toujours codées, s'intégrant dans le système symbolique.

Le cailcedrat, par exemple; est amer, comme est amer le Nya quand on enfreint les règles de bonne conduite qu'il préconise. Le coton, sous forme d'une boule de fil, c'est-à-dire sous sa forme terminale, est le symbole de la perfection, du travail achevé, de la patience.

Le ficus n'toro, associe à la résurrection, à la survie, est un élément important du Nya : des branches de ficus garnissent le toit du Nyagba, le sanctuaire du $N y a$. Les racines du kŭgwe, en bambara n'kǔdye, le premier arbre de la creation selon les récits mythiques, symbolise la durée, la vieillesse du Nya, qui renforce ses pouvoirs. Le süsün, fruit aux grains abondants, symbolise la fécondité de la femme, la progéniture nombreuse. Le "noyau" du yapєre est constitué d'une boule d'or, métal inoxydable considéré comme le symbole de l'intangibilité, de la puissance du Nya. Vendelx mentionne également l'existence d'un aérolythe dans cette partie de l'autel (1).

Un disque de bois représente la terre, que l'on dit située entre sept ciels du haut et sept ciels du bas.

Il ne nous est pas possible d'étudier ici la littérature orale qui se rapporte aux fêtes annuelles du Nya; voici cepenđant quelques expressions désignant le Nya qui permettent dejà de préciser l'idée que s'en font les Minyanka.

(1) Vendeix, Note sur la principale divinite chez les senoufo et les Bambara, in BuZZ. Com. Etudes Historiques et Scien-. tifiques de Z'A.O.F., t. XVI, $\mathrm{n}^{\circ} 1,1933$. 
Parmi les noms donnés au Nya dans les chants et les prières, c'est le terme Mãgala, qui designe chez les Bambara le Dieu suprême, qui revient le pius souvent. Les louanges du Nya proclament : "Dieu (Klє) t'a donne le nom le plus célèbre: Mãgaza. Le Nya apparait comme un principe suprême, redoutable, agressif, "bourdonnant", dont l'âge vénérable force le respect. pour ceux qui "prendraient le mauvais chemin" (de la sorcellerle), il est l'ennemi suprême, le bॄndjugu ou le bendjugu ble, le bॄndjugu rouge, le roúge étant associé à la violence, à la mort. Il est aussi le bama, le bama djugu ou le korofe bamadju$g u$ : le "caiman", le "caĩman redoutable" ou le "redoutable caiman de l'Est", c'est-à-dire de Dieu. "Chose redoutable" (fidjugu), il est aussi "chose redoutable et chose bonne" ( $f \varepsilon d j u g u-f \varepsilon n y u m a)$; selon que l'on respecte ou non les lois ancestrales.

On l'appelle également "colline qui procure les chiens" (tegele pu ta pu we), la colline symbolisant la mort et le chien (associé souvent à la terre et à la mort) symbolisant le sorcier.; ou encore "bonne colline de chiens de minuit tués". Pour les sorciers, les chiens de minuit, le Nya siạnifie la mort. On dit du Nya qu'il est un "voleur de morts" car il dépeuple les maisons des sorciers.

Certaines dénominations du Nya mettent l'accent sur son ancienneté, son âge vénérable : lદgo "vénérable", "vêtéran"; nyє re, nom donné a la première fille d'une femme. Cela signifie non seulement que la société initiatique est la fille d'une société "mère" mais aussi qu'elle est l'ainée, qu'elle a le droit d'ainesse. $B a$ dye signifle "mère blanche" : le Nya est la mère de tous et sa couleur est le blanc, la couleur divine.Plusieurs noms renvoient à la notion de connaissance : on appelle le Nya "notre Bozo", le pêcheur bozo étant le symbole du quide, qui fatt passer le fleuve ; "notre chasseur", car les chasseurs connaissent les "choses de la brousse" et c'est de la brousse que les ancêtres ont rapporté les "bonnes vieilies choses", les yap "vieille femme experte en amour", la jouissance sexuelle êtant symboliquement associée, chez les Minyanka comme chez les 
Bambara (1), a l'accession à la connaissance.

\section{2. sandongo ou}

Sandongo est la hyène, patronne de l'agriculture. Selon les. recits mythiques, elle connait les secrets de la terre et a enseigné les techniques de production aux hommes. Parmi les autels, portatifs du culte figurent la houe (daba) du cultivateur, le harpon (nyદki) du pêcheur, la navette du tisserand ainsi qu'une corne, la "voix de la hyène", qui sert à appeler les cultivateurs au travail. En effet, la confrérie constitue également une société de culture et une société d'entraide. Avant la généralisation de la culture attelée, il y avait en pays minyanka, de véritables "championnats" de culture entre les différentes sociétés, à l'issue desquels les vainqueurs emportaient la corne sacrée des vaincus. Ceux-ci n'avaient alors d'autres recours que de "racheter" la corne, très cher, ou de la reconquérir. Il s'agissait d'être vif comme la hyène afin d'être comparé à elle par les joueurs et les chanteurs qui faisaient les louanges des cultivateurs.

3. Le Manỹa .

-------

Le culte rendu au Manyã est assez rare en pays minyanka. Les autels du Many $\widetilde{a}$ se distinguent par leurs dimensions peu communes : ils peuvent dépasser la taille humaine. Le Manyã est réputé être la "mère" de tous les cultes de possession. Dans la "tête" de ses yapk re, se trouve la racine de la datura kordya, solanacée hallucinogène qui intervient dans le declenchement des scènes du "thếtre sacré" de toutes les grandes sociétés d'initiation minyanka et dont,à l'origine, seul le Many ã avait le secret.

(1) Voir D. Zahan, Sociétés d'initiation bambara, Mouton, 1960. 